\title{
Dissociation and Neurobiological Consequences of Traumatic Stress
}

P. Bob

Department of Psychiatry, $1^{\text {st }}$ Faculty of Medicine, Charles University, Prague

Correspondence to: Petr Bob, Ph.D.

Department of Psychiatry

$1^{\text {st }}$ Faculty of Medicine

Charles University

Ke Karlovu 11

12000 Prague 2

Czech Republic

petrbob@netscape.net

Key words:

dissociation, traumatic stress, epilepsy, kindling

Activitas Nervosa Superior 2008;50:9-14

Abstract

According to recent evidence traumatic stress is an important etiological factor in pathogenesis of psychiatric diseases. Typical psychological manifestation of the traumatic stress represents dissociative states. Dissociated states present pathological conditions where psychological trauma may emerge in a variety of forms such as psychic dissociative symptoms or on the other hand as somatoform symptoms including seizures. At this time there is evidence that temporal lobe seizure activity may produce dissociative syndrome and this activity likely may exist independently of neurological focal lesion. This conceptualization of dissociative phenomena is in accordance with findings that originate from the study of the relationship between epilepsy and mental illness and known as antagonism between epilepsy and psychosis or the so-called forced normalization. From this point of view the relationship between dissociation, epileptic activity and kindling phenomena represent useful concepts for understanding of neurobiological consequences of the traumatic stress and dissociation with respect to pathogenesis of stress-related mental diseases. 


\section{Dissociation and Traumatic Stress}

S cientific history of the dissociation began in the French psychiatry of the 19th century mainly in the work of Pierre Janet. Janet was a follower of many famous psychiatrists or neurologist such as Hughlings Jackson, Jean-Martin Charcot or Theodule Ribot who have meant a great inspiration for his work. Janet comprehensively described many abnormal states of consciousness and studied these states as a defect of the psychic wholeness (Ellenberger, 1970). This means that traumatic or stressful event with extremely negative charge does not fit into current cognitive scheme and is split off from consciousness (van der Hart and Friedman 1989; Putnam, 1997). Dissociation leads to an effort to eliminate these negative memories and increases intrusive thoughts because of contradictory tendencies resulting to inner conflict when unacceptable or traumatic memory is released into the consciousness. According to modern definition, dissociation may be defined as partial or total disconnection between memories of the past, awareness of identity and of immediate sensations, and control of bodily movements often resulting from traumatic experiences, intolerable problems, or disturbed relationships (Colman, 2003). DSM-III-R, DSM-IV and similarly also ICD-10 define dissociation as "a disturbance or alteration in the normally integrative functions of identity, memory or consciousness" (American Psychiatric Association, 1987, 1994; World Health Organization, 1993). In ICD-10 is also defined the organic dissociation, induced by a variety of conditions affecting cerebral function (Good, 1993). Dissociation on the psychic level emerges as memory losses, fragmentation of knowledge of the self and experience, splitting of emotional and cognitive aspects of experiences, numbing of affect, psychological escape from unpleasant stimuli, trance-like states, increased suggestibility and greater hypnotizability (Putnam, 1989, 1997; Hall and Powell, 2000). Dissociative disorders are mainly induced due to a traumatic event. Most often this event represents exposition of a trauma in childhood due to physical, emotional or sexual abuse with following development of symptoms often after many years. Dissociative symptoms also occur due to traumatic events such as accidents and natural disasters. Characteristic features of psychic dissociative symptoms are changes in notion of external world such as derealization, hallucinations, changes of memory predominantly in the form of psychogenic amnestic disorder or changes in notion of identity such as depersonalization or in serious cases multiple personality disorder characterized by distinguished personalities that exist in one person (Putnam, 1989; Hall and Powell, 2000).

Dissociation as a reaction to psychological and/or physical traumatic insult has various neurobiological consequences. One of the first typical reactions are disturbances of selfregulatory systems such as hypothalamus-pituitary-adrenal axis (HPA) resulting in hyperarousal, tachycardia or other symptoms of autonomic nervous system instability. HPA is traditionally understood as stress system of the body which is functionally closely related to neuroendocrinological balance and control hormonal levels, energetic metabolism and neuroimmunomodulation during stress reaction (Teicher et al. 2003; Read, Perry, Moskowitz, and Connoly, 2001). According to neurodevelopmental research are most serious disturbances of HPA axis caused by traumatic events such as childhood abuse or neglect in the first years of life and often have long-term impact on emotional, behavioral, cognitive, social and physiological functions (Putnam, 1997; Ito et al., 1993; Teicher et al. 2003; Read, Perry, Moskowitz, and Connoly, 2001). These neuroendocrinological and neurophysiological dysfunctions as a consequence of the trauma and dissociation are in accordance with recent and historical findings that also somatic components of dissociation have a profound role in the long-term adaptation to traumatic experience and lead to a lack of integration of somatoform components of experience, reactions, and functions (the so-called somatoform dissociation) (Nijenhuis, 2000). Typical symptoms of somatoform dissociation are alterations in sensation of pain (analgesia, kinesthetic anesthesia), painful symptoms, perception alterations, motor inhibition or loss of motor control, gastrointestinal symptoms and dissociative seizures (Nijenhuis et al., 1996; Brown and Trimble 2000; Kuyk et al., 1999).

\section{Dissociation and epileptic discharges}

Relationship of psychological traumatic factors and epilepsy has been mentioned already in the work of famous British neurologist Hughlings Jackson and also by French neurologist Jean-Martin Charcot who for the first time proposed that dissociative symptoms in multiple personality disorder (MPD) are related to epileptic process (Putnam, 1997). Following these findings historical and recent literature describes connection of EEG abnormalities in the patients with MPD. In these studies has been reported that prevalence of seizure disorders is much higher in MPD patients (Mesulam, 1981; Schenk and Bear, 1981; Benson, Miller and Signer, 1986; Perrine, 1991; Putnam, 1997). Mutual relationship between MPD 
and temporal lobe epilepsy support studies that reported epileptic activity during personality alterations in MPD and also identity shift in temporal lobe epilepsy (Schenk and Bear, 1981; Mesulam, 1981; Coons et al., 1982; Benson, Miller, and Signer, 1986; Spiegel, 1991; Hersch, Yiu-Chung, and Smeltzer, 2002). Further reported evidence that describes the relationship between epileptic process and dissociation is based on clinical data that raise a possible role of temporal lobe abnormalities in pathological dissociation (Putnam, 1997; Teicher et al., 2003; Bob, 2003). Frequent EEG abnormalities have been found in traumatized and dissociated patients in non-epileptic conditions and typically involve frontotemporal spikes, sharp waves or paroxysmal slow waves (Putnam, 1997; Teicher et al., 1993, 2003; Ito et al., 1993). Epileptic activity in dissociative states during depersonalization (Sierra and Berrios, 1998), dissociative disorders not otherwise specified, dissociative seizures (the so-called pseudoepilepsy) (Bowman and Coons, 2000) and dissociative states of consciousness such as possession, out of body experiences, near death experiences or religious experiences has also been reported (Saver and Rabin, 1997; Alvarez, 2001; Blanke et al., 2002). On the other hand dissociation-like symptoms such as depersonalization, fugues, amnesias and autoscopy (seeing an externalized image of oneself) are sometimes reported ictally and periictally, by seizure patients (Putnam, 1997). Contemporary data support the evidence that temporallimbic seizure activity can produce dissociative symptoms also without neurological focal lesion (Spiegel, 1991). This evidence linking dissociative symptoms to the temporal-limbic epileptic activity in non-epileptic conditions is consistent with data that the dissociative symptoms in temporal lobe epileptics occur during interictal periods and not during the ictal state (Spiegel, 1991). These findings are in accordance with evidence that in certain psychiatric patients, often without apparent EEG abnormalities, psycho-sensory symptoms of epileptogenic nature occur (the so-called complex partial seizure-like symptoms) (Roberts et al., 1992; Hines et al., 1995). These symptoms, likely closely related to dissociative tendency and experienced traumatic events (Roberts, 1993), normally belong to characteristic manifestations of temporal lobe epilepsy but may also emerge without apparent seizures in the so-called Epilepsy Spectrum Disorders (Roberts et al., 1992; Hines et al., 1995; Jampala et al., 1992). Characteristic symptoms are very similar to certain dissociative symptoms. Typical symptoms are memory gaps, confusion spells, staring spells, episodic irritability, episodic rhinitis, episodic aphasia, jamais-vu, olfactory hallucinations, gustatory hallucinations, visual illusions (e.g., scintillations), paresthesia, anesthesia, auditory illusions (e.g., phone ringing). In addition, the patients suffer from headache with nausea and/or photophobia, abrupt mood shifts, deja-vu, abdominal sensations, intrusive thoughts and parasomnias (Roberts 1992, 1999; Hines et al., 1995). A great many of these symptoms are characteristic in the socalled Epilepsy Spectrum Disorder (ESD). Although the phenomenology of ESD and the positive clinical response to anticonvulsant seen in most ESD patients would suggest the presence of subclinical electrophysiological dysfunction, the lack of clear non-behavioral evidence of CNS dysfunction (i.e., EEG) may obscure the underlying neurological nature of ESD (Roberts, 1992; Hines et al., 1995). The complex partial seizure-like symptoms have also been reported in close relationship to increased sensitivity to parental influence and dissociative tendency related to child abuse and other traumatic or aversive events (Roberts, 1993; Bob et al., 2005).

\section{Epilepsy, kindling and psychopathology}

Above reported findings suggest the hypothesis that traumatic stress similarly as other traumatic insults might induce a hyper-excitable state linked to a lowered seizure threshold and can lead to a latent epileptogenic process or may result in epilepsy. The kindling model of epilepsy has modeled these processes in animals. Kindling is actually the repetition of sub-convulsive electric stimuli that can induce lowered seizure threshold and may result in a progressive epileptic state. Although this model is quite elaborated in animals it is unclear whether kindling really corresponds to human epileptogenesis (Jensen and Baram, 2000; Walker et al., 2002). The kindling model has also been proposed as an explanation for the relationship between epileptic-like phenomena related to repeated trauma and stress, such as child abuse and neglect (Post, Weis, and Smith, 1995; Putnam, 1997; Teicher et al., 2003).

There are good reasons for using limbic kindling as a model in epileptogenesis of focal human limbic epilepsy or complex partial seizures with secondary generalization (Adamec, 1990; Albright and Burnham, 1980; Loscher et al., 1986). This notion is supported by findings (Adamec, 1990; Monroe 1982) of repeated electrical stimulation in human hippocampus that led to an epileptic disorder, which was not present before the experiment and there are other reported cases of kindling in thalamus (Adamec, 1990; Sramka et al., 1977). This notion is not clear evident because there is not many reported cases of kindling in humans. But there is another reason for this 
notion, which consists in the evidence that timedependent spread of epileptic excitability occurs independently on tissue pathology as a consequence of organic damages (Adamec, 1990). According to some findings (Adamec, 1990) damage creates kindling stimulus that leads to a seizure disorder and the delay between trauma and onset of seizures in humans is consistent with these findings (Adamec, 1990). These findings are supported also the successful prophylactic anticonvulsant therapy following head trauma with neurological signs of brain damage that reduce the incidence of an epileptic disorder development (Adamec, 1990; Servit, Musil 1981).

Kindling may be also used at the general level of explanation of psychopathology as a mirror of altered limbic functions. In manic depressive psychosis the role of kindling was hypothesized in the study of influence of carbamazepin on mood and its anticonvulsant and antikindling effect (Adamec, 1990). It corresponds to the anticonvulsant effect of carbamazepin in complex partial seizures (Adamec, 1990) and also its influence on mood of these patients. It led to the notion that effectiveness of carbamazepin in the treatment of manic depressive disorder in nonepileptics is due to its limbic anticonvulsant properties with the hope of understanding limbic neural mechanism underlying manic depressive disorder (Adamec, 1990; Dalby, 1975). Dopaminergic hypothesis of schizophrenia provides similar results that show schizophrenic symptoms as consequences of hyperdopaminergic kindling in mesolimbic dopaminergic system (Adamec, 1990). Some findings also point to the effect of limbic seizures on dopaminergic functions (Adamec, 1990) and as written above there is a relationship of reciprocity between that kindling in mesolimbic dopaminergic and similar EEG activity in temporal neocortex in patients with temporal lobe epilepsy (Pakalnis et al., 1988). The concept of kindling as a model for psychopathology is also in accordance with recent findings that schizophrenia as well as depression are related to a loss of physiological balance between excitation and inhibition. The significant loss of physiological equilibrium is observed also in epilepsy which is linked to over-excitation while on the other hand schizophrenia or depression are likely connected to overinhibition in the structures of the limbic system. In epilepsy, the normal equilibrium between excitation and inhibition permanently alters by repeated focal excitation or kindling, resulting in a permanent state of excessive focal excitability and spontaneous seizures (Stevens 1999; Goddard, McIntyre, and Leech, 1969). Recent findings indicate that similar "kindling" or sensitization may originate in inhibitory systems in response to focal physiological pulsed discharges of limbic and hypothalamic neurons and this excess of inhibitory factors may then manifest as a psychosis (Stevens, 1992, 1999). Similar situation is also in depression because decreased activity of serotonin, norepinephrine, dopamine, and GABA may facilitate the kindling process (Kanner and Balabanov, 2002). In this context Flor-Henry found that psychotic patients with predominantly schizophrenic symptoms had a high incidence of epileptic foci in the left temporal lobe while depressive patients had a high incidence of foci in the right temporal lobe (Flor-Henry, 1976, 2003; Hugdahl, 2001). This suggests an association between unilateral hemispheric dysfunction and ipsilateral temporal-limbic epileptic focus (Flor-Henry, 1976, 2003; Shulman, 2003; Hugdahl, 2001). These findings in principle agree with reported cases of forced normalization or alternative psychosis in which decreasing of epileptic symptomatology is linked to increased psychopathology in the form of psychosis or depression and on the contrary (Jobe et al., 1999; Wolf and Trimble, 1985; Krishnamoortthy et al., 2002).

\section{Conclusion}

The kindling in inhibitory systems might explain occurrence of complex partial seizure-like symptoms in psychiatric patients because of close relationship of traumatic stress, dissociation and complex partial seizurelike symptoms. The findings also suggest the relevance of the kindling model of dissociative states as a consequence of repeated traumatic stress (Post et al., 1995; Putnam, 1997 ) in various psychiatric diagnoses for the explanation of neurobiological mechanism of repeated traumatic stress in etiopathogenesis of psychiatric disorders. The findings regarding influence of traumatic stress therefore represent useful and testable concepts for future research that may substantially illuminate neurobiological processes underlying generation of typical psychiatric symptoms in certain mental diseases as well as in several epileptic syndromes.

This study was supported by grants MSM002160849, MSM0021622404, by the research project of Centre for Neuropsychiatric Research of Traumatic Stress 1 M06039.

\section{REFERENCES}

Adamec RE (1990). Does kindling model anything clinically relevant? Biol Psychiatry, 27, 249-79. 
Albright PS and Burnham BM (1980). Development of a new pharmacological seizure model: Effects of anticonvulsants on cortical and amygdala-kindled seizures in the rats. Epilepsia, 21, 681-689.

Alvarez J (2001). Neural hypersynchronization, creativity and endogenous psychoses. Med Hypotheses 56: 672-685.

American Psychiatric Association (1987). DSM III-R, Diagnostic and Statistical Manual of Mental Disorders, fourth ed. Washington DC: American Psychiatric Association.

American Psychiatric Association (1994). DSM IV, Diagnostic and Statistical Manual of Mental Disorders, fourth ed. Washington DC: American Psychiatric Association.

Benson F, Miller BL and Signer SF (1986). Dual personality associated with epilepsy. Arch Neurol 43: 471-474.

Blanke O, Ortigue S, Landis T, Seeck M (2002). Neuropsychology: Stimulating illusory own-body perceptions. Nature 419: 269-270.

Bob P (2003). Dissociation and Neuroscience: History and New Perspectives. Int J Neurosci. 113: 903-914.

Bob P, Susta M, Pavlat J, Hynek K, Raboch J (2005). Depression, traumatic dissociation and epileptic-like phenomena. Neuro Endocrinol Lett. 26: 321-325.

Bowman ES and Coons PM (2000). The differential diagnosis of epilepsy, pseudoseizures, dissociative identity disorder, and dissociative disorder not otherwise specified. Bull Menninger Clin, $64,164-180$

Brown RJ and Trimble MR (2000). Dissociative psychopathology, non-epileptic seizures, and neurology. J Neurol Neurosurg Psychiatr 69: 285-9.

Colman AM (2003). A Dictionary of Psychology. New York: Oxford University Press.

Coons PM, Milstein V and Marley C (1982). EEG studies of two multiple personality and a control. Arch Gen Psychiatry 39: 823-825.

Dalby MA (1975). Behavioral effects of carbamazepine. In: Complex partial seizures and their treatment, Advances in Neurology vol II. Penry J.K., Daly D.D. (eds), Raven Press, New York, 331-343.

Ellenberger HF (1970). The Discovery of the Unconscious: The History and Evolution of Dynamic Psychiatry. New York: Basic.

Flor-Henry P (2003). Lateralized temporal-limbic dysfunction and psychopatology. Epilepsy Behav, 4, 578-590.

Goddard GV, Mclntyre GC and Leech CK (1969). A permanent change in brain function resulting from daily electrical stimulation. Exp Neurol. 25: 295-330.

Good MI (1993). The Concept of an Organic Dissociative Syndrome: What is the Evidence? Harv Rev Psychiatry 1: 145-57.

Hall JM and Powell J (2000). Dissociative Experiences Described by Women Survivors of Childhood Abuse. J Interpers Violence, 15, 184204
Hines M, Swan C, Roberts RJ, Varney NR (1995). Characteristics and mechanisms of epilepsy spectrum disorder: An explanatory model. Appl Neuropsychol. 2: 1-6.

Hugdahl K (2001). Psychophysiology, The Mind-Body Perspective. Cambridge: Harvard University Press.

Ito Y, Teicher M, Gold C, Harper D, Magnus E, Gelbard H (1993). Increased prevalence of electrophysiological abnormalities in children with psychological, physical and sexual abuse. J Neuropsychiatry Clin Neurosci. 5: 401-408.

Jampala V, Atre-Vaidya $\mathbf{N}$ and Taylor MA (1992). A profile of psychomotor symptoms (POPS) in psychiatric patients. Neuropsychiatry Neuropsychol Behav Neurol. 5: 15-19.

Jensen FE and Baram TZ (2000). Developmental seizures induced by common early-life insults: short- and long-term effects on seizure susceptibility. Mental retardation and developmental disabilities research reviews, 6, 253-7.

Jobe PC, Dailey JW and Wernicke JF (1999). A noradrenergic and serotoninergic hypothesis of the linkage between epilepsy and affective disorders. Crit Rev Neurobiol. 13: 317-356.

Kanner AM and Balabanov A (2002). Depression and Epilepsy. How closely related are they? Neurology 58: 827-839.

Krishnamoorthy ES, Trimble MR, Sander JW, Kanner AM (2002). Forced normalization at the interface between epilepsy and psychiatry. Epilepsy Behav. 3: 303-308.

Kuyk J, Spinhoven P, Van Emde Boas W, Van Dyck R (1999). Dissociation in Temporal Lobe Epilepsy and Pseudo-Epileptic Seizure Patients. J Nerv Ment Dis. 187: 713-720.

Loscher W, Jackel R, Czucvar J (1986). Is amygdala kindling in rats a model for drug-resistant partial epilepsy? Exp Neurol, 93, 211-226. Mesulam MM (1981). Dissociative states with abnormal temporal lobe EEG. Arch Neurol. 38: 176-181.

Monroe RR (1982). Limbic Ictus and Atypical Psychoses. J Nerv Ment Dis. 170: 711-716.

Nijenhuis ERS (2000). Somatoform dissociation: major symptoms of dissociative disorders. J Trauma Dissociation, 1, 7-32.

Nijenhuis ERS, Spinhoven P, Van Dyck R, Van Der Hart 0 and Vanderlinden J (1996). The development and psychometric characteristics of the somatoform dissociation questionnaire (SDQ20). J Nerv Ment Dis, 184, 688-694.

Pakalnis A, Drake ME, Kuruvilla J, Blake JK (1988). Forced normalization. Arch Neurol. 45: 139.

Perrine KR (1991). Psychopathology in epilepsy. Semin Neurol. 11: 175-181.

Post RM, Weis SR and Smith MA (1995). Sensitization and kindling. In: Friedman MJ, Charney DS, Deutch AY, editors. Neurobiological and clinical consequences of stress: From normal adaptation to posttraumatic stress disorder. Philadelphia: Lipincott-Raven.

Putnam FW (1989). Diagnosis and Treatment Multiple Personality Disorder, The Guilford Press, New York, London. 
Putnam F (1997). Dissociation in Children and adolescents. A developmental Perspective. London, New York: The Guilford Press Read J, Perry BD, Moskowitz A, Connolly J (2001). The contribution of early traumatic events to schizophrenia in some patients: a traumagenic neurodevelopmental model. Psychiatry 64: 319-45.

Roberts RJ, Gorman LL, Lee GP, Hines ME, Richardson ED, Riggle TA, Varney NR (1992). The phenomenology of multiple partial seizure like symptoms without stereotyped spells: An epilepsy spectrum disorder? Epilepsy Res. 13: 167-177.

Roberts RJ (1993). Commentary; Positive associations among dichotic listening errors, complex partial epileptic-like signs, and paranormal beliefs. J Nerv Ment Dis. 131: 668-671.

Saver JL and Rabin J (1997). The neural substrates of religious experience. J Neuropsychiatry Clin Neurosci. 9: 498-510.

Schenk L and Bear D (1981). Multiple personality and related dissociative phenomena in patients with temporal lobe epilepsy. Am J Psychiatry 138: 1311-1316.

Servit Z and Musil F(1981). Prophylactic treatment of posttraumatic epilepsy: results of a long-term follow-up in Czechoslovakia. Epilepsia, 22, 315-320.

Shulman MB (2003). Laterality and psychopathology circa 1976. Epilepsy Behav, 4, 576-577.

Sierra M and Berrios GE (1998). Depersonalization: neurobiological perspectives. Biol Psychiatry, 44, 898-908.

Spiegel D (1991). Neurophysiological correlates of hypnosis and dissociation. J Neuropsychiatry Clin Neurosci. 3: 440-5.

Spiegel D and Cardena E (1991). Disintegrated Experience: The Dissociative Disorders Revisited. J Abnorm Psychol. 100: 366-376.

Sramka M, Sedlak P, and Nadvornik P (1977). Observation of kindling phenomenon in treatment of pain by stimulation in thalamus. In: Neurosurgical treatment in psychiatry. Sweet W (ed), Baltimore: University Park Press, p. 651-654.

Stevens JR (1992). Abnormal reinnervation as a basis for schizophrenia: a hypothesis. Arch Gen Psychiatry, 49, 238-243.

Stevens JR (1999). Epilepsy, schizophrenia and the extended amygdala. Ann NY Acad Sci.: 156: 548-561.

Teicher M, Glod C, Surrey J, Swett C (1993). Early childhood abuse and limbic system ratings in adult psychiatric outpatients. J Neuropsychiatry Clin Neurosci. 5: 301-306.

Teicher M, Andersen SL, Polcari A, Anderson CM, Navalta CP, Dennis M, Kim DM (2003). The neurobiological consequences of early stress and childhood maltreatment. Neurosci Biobehav Rev. 27: 3-44.

van der Hart O and Friedman B (1989). A Reader's Guide to Pierre Janet on Dissociation: A Neglected Intelectual Heritage. Dissociation 2: 3-16.

Walker MC, White HS, and Sander JW (2002). Disease modification in partial epilepsy. Brain, 125, 1937-50.

Wolf P and Trimble MR (1985). Biological Antagonism and Epileptic Psychosis. Br J Psychiatry 146: 72-276.
World Health Organization (1993). The ICD-10. Classification of Mental and Behavioural Disorders. Diagnostic Criteria for Research. Geneva: World Health Organization. 